\title{
MULTI-OBJECTIVE OPTIMIZATION USING LOCAL FRACTIONAL DIFFERENTIAL OPERATOR
}

\author{
RABHA W. IBRAHIM ${ }^{1, *}$, MASLINA DARUS ${ }^{2}$ \\ ${ }^{1}$ Cloud computing center, University of Malaya, Malaysia \\ ${ }^{2}$ Centre of Modelling and Data Sciences, Faculty of Science and Technology, \\ Universiti Kebangsaan Malaysia, 43600 UKM Bangi, Selangor, Malaysia \\ *Corresponding author: rabhaibrahim@yahoo.com
}

\begin{abstract}
In this effort, we aim to generalize the concept of Univex functions by utilizing a local fractional differential-difference operator, based on different types of local fractional calculus (fractal calculus). This study leads to a new class of these functions in some optimal problems by illustrating conditions on the generalized functions. We call it the class of local fractional Univex functions. Strong, weak, converse, and strict converse duality theorems are given. Multi-objective optimal problem involves the new process is solved (local optimal problem). The main tool employed in the analysis is based on the local fractional derivative operators.
\end{abstract}

\section{INTRODUCTION}

The notion of local fractional calculus (also labeled fractal calculus), which was first suggested by Kolwankar and Gangal [1] using the Riemann-Liouville fractional derivative [2]. It was employed to deal with non-differentiable issues from science and engineering [3]- [5]. Local fractional derivative of $\phi(\chi)$ of order $0<\alpha \leq 1$ is specified by

Received 2018-09-24; accepted 2018-11-20; published 2019-03-01.

2010 Mathematics Subject Classification. 44A45.

Key words and phrases. fractional calculus; fractional operator; fractional differential equation; univex function.

(C)2019 Authors retain the copyrights of their papers, and all open access articles are distributed under the terms of the Creative Commons Attribution License. 


$$
D^{\alpha} \phi(\chi)=\left.\frac{d^{\alpha} \phi(\chi)}{\chi^{\alpha}}\right|_{\chi=\chi_{0}}=\lim _{\chi \rightarrow \chi_{0}} \frac{d^{\alpha}\left[\phi(\chi)-\phi\left(\chi_{0}\right)\right]}{\left[d\left(\chi-\chi_{0}\right)\right]^{\alpha}},
$$

where the expression $d^{\alpha}\left[\phi(\chi)-\phi\left(\chi_{0}\right)\right] /\left[d\left(\chi-\chi_{0}\right)\right]^{\alpha}$ is the Riemann-Liouville fractional derivative given by

$$
\frac{d^{\alpha} \phi(\chi)}{d \chi^{\alpha}}=\frac{1}{\Gamma(1-\alpha)} \frac{d}{d \chi} \int_{0}^{\chi} \frac{\phi(t)}{(\chi-t)^{\alpha}} d t
$$

corresponding to the integral operator

$$
\left(I^{\alpha} \phi\right)(\chi)=\frac{1}{\Gamma(\alpha)} \int_{0}^{\chi}(\chi-t)^{\alpha-1} \phi(t) d t
$$

This operator is well-defined and it is represented to the classical fractional calculus.

The local fractional derivative utilizing the fractal geometry is defined by the formula [5]

$$
D^{\alpha} \phi(\chi)=\left.\frac{d^{\alpha} \phi(\chi)}{\chi^{\alpha}}\right|_{\chi=\chi_{0}}=\lim _{\chi \rightarrow \chi_{0}} \frac{\Delta^{\alpha}\left[\phi(\chi)-\phi\left(\chi_{0}\right)\right]}{\left[\left(\chi-\chi_{0}\right)\right]^{\alpha}}
$$

where

$$
\Delta^{\alpha}\left[\phi(\chi)-\phi\left(\chi_{0}\right)\right] \cong \Gamma(\alpha+1)\left[\phi(\chi)-\phi\left(\chi_{0}\right)\right]
$$

Dunkl operator (see $[6,7])$ is a structure for a diff-difference operator

$$
\Lambda_{\kappa} \phi(\chi)=\frac{d}{d \chi} \phi(\chi)+\kappa\left(\frac{\phi(\chi)-\phi(-\chi)}{2 \chi}\right), \quad \kappa \geq 0 .
$$

It generalized some special functions and integral transforms in several variables connected with reflection groups. This class of operators has developed many other operators. It applied in the analysis of quantum many body systems. Recently, this operator is given in term of fractional calculus [8]. By employing the local fractional differential operator in (1.1) or (1.2), we introduce a generalization of (1.3) as follows:

$$
\begin{gathered}
\Lambda_{\kappa}^{\alpha} \phi(\chi)=D^{\alpha} \phi(\chi)+\kappa\left(\frac{\phi(\chi)-\phi(-\chi)}{2 \chi}\right), \\
(\kappa \geq 0, \alpha \in(0,1], \chi \neq 0 \in \mathbb{R})
\end{gathered}
$$

In this study, we aim to generalize the concept of Univex functions by utilizing a local fractional differentialdifference operator (1.4). This study leads to a new class of these functions in some optimal problems by illustrating conditions on the generalized functions. We call it the class of local fractional Univex functions. Strong, weak, converse, and strict converse duality theorems are given, with examples in the sequel. 


\section{UNIVEX FUNCTION}

In this section, we generalize the concept of the Univex function, by using the local fractional Dunkl operator. Define the following functions $\eta:[a, b] \times[a, b] \rightarrow \mathbb{R} \backslash\{0\}, \phi:[a, b] \rightarrow \mathbb{R}$ and $\Phi: \mathbb{R} \rightarrow \mathbb{R}$.

Definition 1. A differential function $\phi$ is said to be a local fractional univex function of order $\alpha \in(0,1]$ in the direction of $\xi \in J:=[a, b]$ if for all $\chi \in J$, we have

$$
\Lambda_{\kappa}^{\alpha} \phi(\chi) \leq \frac{\Phi(\phi(\chi)-\phi(\xi))}{\eta(\chi, \xi)} .
$$

Note that, this concept is one of significant tool for optimization. Also, we confirm that there are many other techniques for optimization which are generalized by fractional formal operators (see [9]- [12]). The advantage of using the fractional Dunkl operator, is that can be acted on multi-dimensional Euclidean spaces. Therefor, it can be employed in non-linear multi-objective problem

$$
\begin{array}{ll}
\text { Minimize } & \Psi(\chi)=\left(\psi_{1}(\chi), \ldots, \psi_{n}(\chi)\right) \\
\text { subject to } & \Theta(\chi) \leq 0
\end{array}
$$

where $\Psi: J \rightarrow \mathbb{R}^{n}$ and $\Theta: J \rightarrow \mathbb{R}^{n}$ and 0 is the zero vector in $\mathbb{R}^{n}$. The function $\Psi(\chi)$ has many applications in various studies. It may represent a multi-agent function in cloud computing systems.

Definition 2. A point $\xi \in J:=\{\chi \in J: \Theta(\chi) \leq 0\}$ is said to be an efficient outcome of (2.1), if there is no point $\chi \in J$, with $\Psi(\chi) \leq \Psi(\xi)$. Moreover, it is known as a weak efficient outcome when $\Psi(\chi)<\Psi(\xi)$.

Definition 3. The couple $(\Psi, \Theta)$ is called a local fractional univex of order $\alpha$, if for all $\chi \in J$ we have

$$
\eta_{1}(\chi, \xi) \cdot \mathfrak{D}_{\kappa}^{\alpha} \Psi(\chi) \leq \phi_{1}(\Psi(\chi)-\Psi(\xi))
$$

and

$$
\eta_{2}(\chi, \xi) \cdot \mathfrak{D}^{\alpha} \Theta(\chi) \leq-\phi_{2}(\Theta(\chi)-\Theta(\xi))
$$

where

$$
\eta_{1}: J \times J \rightarrow \mathbb{R}^{n}, \eta_{2}: J \times J \rightarrow \mathbb{R}^{n}, \phi_{1}: \mathbb{R}^{n} \rightarrow \mathbb{R}, \phi_{2}: \mathbb{R}^{n} \rightarrow \mathbb{R}
$$

and

$$
\mathfrak{D}_{\kappa}^{\alpha} \Psi(\chi)=\left(\Lambda_{\kappa}^{\alpha} \psi_{1}(\chi), \ldots, \Lambda_{\kappa}^{\alpha} \psi_{n}(\chi)\right)
$$

This class of local fractional univex functions is denoted by $\alpha$-type univex. 


\section{Results}

In this section, we investigate some sufficient optimality conditions for a point to be an efficient solution of (1.3) under the generalized $(\alpha, \rho, \eta, \vartheta)$-type Univex.

Theorem 3.1. Let $\xi$ be an initial solution of the multi-objective problem (1.3) and $c_{1}$ and $c_{2}$ be two nonnegative constants such that

(A) $\Theta(\xi)=0$;

(B) $c_{1}\left(\eta_{1}(x, \xi) \cdot \mathfrak{D}^{\alpha} \Psi(x)\right)+c_{2}\left(\eta_{2}(x, \xi) \cdot \mathfrak{D}^{\alpha} \Theta(x)\right) \geq 0$;

(C) The couple $(\Psi, \Theta)$ is a strong (or weak) pseudo-quasi $(\alpha, \rho, \eta, \vartheta)$-type univex at $\xi \in \Omega$;

(D) $u \leq 0 \in \mathbb{R}^{m} \Rightarrow \phi_{1}(u) \leq 0$ and $v \geq 0 \in \mathbb{R}^{p} \Rightarrow \phi_{2}(v) \geq 0$;

(E) $c_{1} \rho_{1}+c_{2} \rho_{2} \geq 0$.

Then $\xi$ is an efficient solution of (1.3).

Proof. Suppose that $\xi$ is not an efficient solution of (1.3), then there exists $x \in \Lambda$ such that $\Psi(x) \leq \Psi(\xi)$. By the assumptions (A) and (D), we have

$$
\phi_{1}(\Psi(x)-\Psi(\xi)) \leq 0, \quad \text { and } \quad \phi_{2}(\Theta(\xi)) \geq 0 .
$$

In view of the assumption $(\mathrm{C})$, we get

$$
c_{1}\left(\eta_{1}(x, \xi) \cdot \mathfrak{D}^{\alpha} \Psi(x)\right)<-c_{1} \rho_{1}\|\vartheta(x, \xi)\|^{2}
$$

and

$$
c_{2}\left(\eta_{2}(x, \xi) \cdot \mathfrak{D}^{\alpha} \Theta(x)\right) \leq-c_{2} \rho_{2}\|\vartheta(x, \xi)\|^{2} .
$$

Summing the above inequalities and utilizing (E), we conclude that

$$
\begin{aligned}
c_{1}\left(\eta_{1}(x, \xi) \cdot \mathfrak{D}^{\alpha} \Psi(x)\right)+c_{2}\left(\eta_{2}(x, \xi) \cdot \mathfrak{D}^{\alpha} \Theta(x)\right) & <-\left(c_{1} \rho_{1}+c_{2} \rho_{2}\right)\|\vartheta(x, \xi)\|^{2} \\
& \leq 0,
\end{aligned}
$$

which contradicts the assumption (B). Hence, $\xi$ is an efficient solution of (1.3). This completes the proof.

Theorem 3.2. If the following conditions are satisfied:

(A) $\xi$ is a weakly efficient solution of (1.3);

(B) $\Theta$ is continuous in $\xi$;

(C) The functions $\Psi$ and $\Theta$ are fractional Univex functions of order $\alpha \in(0,1)$ in the direction of $\xi \in \Lambda$. Moreover, for some $\bar{x} \in \Lambda$, we have $\Theta(\bar{x})<0$. 
Then there are two constants $c_{1} \geq 0$ and $c_{2} \geq 0$ such that

$$
\begin{gathered}
c_{1}\left(\eta_{1}(x, \xi) \cdot \mathfrak{D}^{\alpha} \Psi(x)\right)+c_{2}\left(\eta_{2}(x, \xi) \cdot \mathfrak{D}^{\alpha} \Theta(x)\right) \geq 0 \\
\left(x \in \Omega, c_{2} \Theta(\xi)=0, \eta_{1}: \Omega \times \Omega \rightarrow \mathbb{R}^{m}, \eta_{2}: \Omega \times \Omega \rightarrow \mathbb{R}^{p}\right) .
\end{gathered}
$$

Proof. Our aim is to show that the system

$$
\eta_{1}(x, \xi) \cdot \mathfrak{D}^{\alpha} \Psi(x)<0, \quad \eta_{2}(x, \xi) \cdot \mathfrak{D}^{\alpha} \Theta(x)<0,
$$

has no solution for $x \in \Omega$. Let the system has a solution $y \in \Omega$. By the assumption (A), we have

$$
\Psi\left(\xi+\epsilon_{1} y\right)<\Psi(\xi) \text { and } \Theta\left(\xi+\epsilon_{2} y\right)<\Theta(\xi)
$$

for sufficient small arbitrary constants $\epsilon_{1}, \epsilon_{2}>0$. Now, we let $\bar{x}:=\xi+\epsilon_{2} y$; which implies that $\bar{x} \in \Lambda \cap N_{\epsilon_{2}}(\xi)$ thus by $(\mathrm{B})$ and $(\mathrm{C})$, we have $\Theta\left(\xi+\epsilon_{2} y\right)=\Theta(\bar{x})<0$; which contradicts $(\mathrm{A})$, where $\xi$ is a weak solution. Therefore, the above inequalities are non-negative. Hence, in view of (C) these are two constants $c_{1}$ and $c_{2}$ satisfy the inequality

$$
c_{1}\left(\eta_{1}(x, \xi) \cdot \mathfrak{D}^{\alpha} \Psi(x)\right)+c_{2}\left(\eta_{2}(x, \xi) \cdot \mathfrak{D}^{\alpha} \Theta(x)\right) \geq 0
$$

with the property $c_{2} \Theta(\xi)=0$. This completes the proof.

Next, we consider the dual problem of (1.3) as follows:

$$
\begin{gathered}
\operatorname{Max} \Psi(\chi)=\left(\psi_{1}(\chi), \ldots, \psi_{m}(\chi)\right) \\
\text { subject to } \quad c_{1}\left(\eta_{1}(x, \chi) \cdot \mathfrak{D}^{\alpha} \Psi(x)\right)+c_{2}\left(\eta_{2}(x, \chi) \cdot \mathfrak{D}^{\alpha} \Theta(x)\right) \geq 0 \\
c_{2} \Theta(\chi) \geq 0
\end{gathered}
$$

where $\chi \in \Omega, c_{1}$ and $c_{2}$ be two non negative constants.

Theorem 3.3. Let $x, \chi$ be initial solutions of the multi-objective problems (1.3) and (3.2) respectively. If

(A) The couple $(\Psi, \Theta)$ is a strong (or weak) pseudo-quasi $(\alpha, \rho, \eta, \vartheta)$-type univex at $\xi \in \Omega$;

(B) $u \leq 0 \in \mathbb{R}^{m} \Rightarrow \phi_{1}(u) \leq 0$ and $v \geq 0 \in \mathbb{R}^{p} \Rightarrow \phi_{2}(v) \geq 0$;

(C) $c_{1} \rho_{1}+\rho_{2} \geq 0$;

then $\Psi(x) \not \Psi(\chi)$.

Proof. Suppose that $\Psi(x) \leq \Psi(\chi)$. Since $c_{1} \rho_{1}+\rho_{2} \geq 0$ then by $(\mathrm{B})$, we obtain

$$
\begin{aligned}
& \phi_{1}(\Psi(x)-\Psi(\chi)) \leq 0 \\
& \phi_{2}(\Theta(\chi)) \geq 0
\end{aligned}
$$


In virtue of the assumption (A) the above inequalities yield

$$
\begin{aligned}
& \left(\eta_{1}(x, \chi) \cdot \mathfrak{D}^{\alpha} \Psi(\chi)\right)<-\rho_{1}\|\vartheta(x, \chi)\|^{2} \\
& \left(\eta_{2}(x, \chi) \cdot \mathfrak{D}^{\alpha} \Theta(\chi)\right) \leq-\rho_{2}\|\vartheta(x, \chi)\|^{2}
\end{aligned}
$$

consequently, we obtain

$$
c_{1}\left(\eta_{1}(x, \xi) \cdot \mathfrak{D}^{\alpha} \Psi(x)\right)<-c_{1} \rho_{1}\|\vartheta(x, \chi)\|^{2}
$$

and

$$
c_{2}\left(\eta_{2}(x, \chi) \cdot \mathfrak{D}^{\alpha} \Theta(x)\right) \leq-\rho_{2}\|\vartheta(x, \chi)\|^{2} .
$$

Summing the above inequalities and utilizing (C), we conclude that

$$
\begin{aligned}
c_{1}\left(\eta_{1}(x, \chi) \cdot \mathfrak{D}^{\alpha} \Psi(\chi)\right)+c_{2}\left(\eta_{2}(x, \chi) \cdot \mathfrak{D}^{\alpha} \Theta(\chi)\right) & <-\left(c_{1} \rho_{1}+\rho_{2}\right)\|\vartheta(x, \chi)\|^{2} \\
& \leq 0,
\end{aligned}
$$

which contradicts the assumption (C). This completes the proof.

Theorem 3.4. Let $x_{0}$ and $\chi_{0}$ be initial solution for the problems (1.3) and (3.2) respectively. If $\Psi\left(x_{0}\right)=$ $\Psi\left(\chi_{0}\right)$ then the (weak or strong) duality problems (1.3) and (3.2) has efficient solutions $x_{0}$ and $\chi_{0}$ respectively.

Proof. Suppose that $x_{0}$ is not efficient for (1.3), then for some $x \in \Lambda$

$$
\Psi(x) \leq \Psi\left(x_{0}\right)=\Psi\left(\chi_{0}\right)
$$

which contradicts weak (strong) duality theorems as $\chi_{0}$ is initial solution for (3.2). Therefore, $x_{0}$ is efficient for (1.3). Similarly $\chi_{0}$ is efficient solution for (3.2). Hence the proof.

Theorem 3.5. Let $\chi_{0}$ be an initial solution of the multi-objective problem (3.2) and $c_{1}$ and $c_{2}$ be two non negative constants such that

(A) The couple $(\Psi, \Theta)$ is a strong (or weak) pseudo-quasi $(\alpha, \rho, \eta, \vartheta)$-type univex at $\xi \in \Omega$;

(B) $u \leq 0 \in \mathbb{R}^{m} \Rightarrow \phi_{1}(u) \leq 0$ and $v \geq 0 \in \mathbb{R}^{p} \Rightarrow \phi_{2}(v) \geq 0$;

(C) $c_{1} \rho_{1}+\rho_{2} \geq 0$.

Then $\chi_{0}$ is an efficient solution of (3.2).

Proof. Suppose that $\chi_{0}$ is not an efficient solution of (3.2), then there exists $x_{0} \in \Lambda$ such that $\Psi\left(x_{0}\right) \leq \Psi\left(\chi_{0}\right)$. Now going on as in Theorem 3.3, we have a contradiction. Hence, $\chi_{0}$ is an efficient solution of (3.2).

Theorem 3.6. Let $x_{0}, \chi_{0}$ be initial solutions of the multi-objective problems (1.3) and(3.2) respectively. If

(A) $\Psi\left(x_{0}\right) \leq \Psi\left(\chi_{0}\right)$;

(B) The couple $(\Psi, \Theta)$ is a strong (or weak) pseudo-quasi $(\alpha, \rho, \eta, \vartheta)$-type univex at $\xi \in \Omega$;

(C) $u \leq 0 \in \mathbb{R}^{m} \Rightarrow \phi_{1}(u) \leq 0$ and $v \geq 0 \in \mathbb{R}^{p} \Rightarrow \phi_{2}(v) \geq 0$; 
(D) $c_{1} \rho_{1}+\rho_{2} \geq 0$

then $x_{0}=\chi_{0}$.

Proof. Suppose that $x_{0} \neq \chi_{0}$. Since $\chi_{0}$ is an initial solution for (3.2) then by (A) and (C), we have

$$
\begin{aligned}
& \phi_{1}\left(\Psi\left(x_{0}\right)-\Psi\left(\chi_{0}\right)\right) \leq 0 \\
& \phi_{2}\left(\Theta\left(\chi_{0}\right)\right) \geq 0 .
\end{aligned}
$$

In virtue of the assumption (B) the above inequalities imply that

$$
\begin{aligned}
& \left(\eta_{1}\left(x_{0}, \chi_{0}\right) \cdot \mathfrak{D}^{\alpha} \Psi\left(\chi_{0}\right)\right)<-\rho_{1}\left\|\vartheta\left(x_{0}, \chi_{0}\right)\right\|^{2} \\
& \left(\eta_{2}\left(x_{0}, \chi_{0}\right) \cdot \mathfrak{D}^{\alpha} \Theta\left(\chi_{0}\right)\right) \leq-\rho_{2}\left\|\vartheta\left(x_{0}, \chi_{0}\right)\right\|^{2},
\end{aligned}
$$

which on summing yields

$$
\begin{aligned}
c_{1}\left(\eta_{1}\left(x_{0}, \chi_{0}\right) \cdot \mathfrak{D}^{\alpha} \Psi\left(\chi_{0}\right)\right)+c_{2}\left(\eta_{2}\left(x_{0}, \chi_{0}\right) \cdot \mathfrak{D}^{\alpha} \Theta\left(\chi_{0}\right)\right) & <-\left(c_{1} \rho_{1}+\rho_{2}\right)\left\|\vartheta\left(x_{0}, \chi_{0}\right)\right\|^{2} \\
& \leq 0,
\end{aligned}
$$

which contradicts to initially of $\chi_{0}$. Then we obtain $x_{0}=\chi_{0}$. This completes the proof.

\section{Simulation}

In this section, we illustrate a simulation to show how the fractional calculus is effected on the multiobjective functions.

Let $\Psi, \Theta: \mathbb{R} \rightarrow \mathbb{R}^{2}$ such that

$$
\Psi(x)=\left(x^{2}, x^{3}\right) ; \quad \Theta(x)=\left(x, x^{2}\right) .
$$

Our aim is to show that the couple $(\Psi, \Theta)$ is $(\alpha, \rho, \eta, \vartheta)$-type univex at $\xi \in[0,1]$. To determine the fractional Dunkl operator on these functions, we shall introduce three cases depending on the value of $k_{v}$ for $v=1$.

4.1. Case (i) $k_{v}=0$. The fractional Dunkl operator acts on the functions $\Psi$ and $\Theta$ as follows:

$$
\mathfrak{D}^{\alpha} \Psi(x)=\left(\frac{\Gamma(3)}{\Gamma(3-\alpha)} x^{2-\alpha}, \frac{\Gamma(4)}{\Gamma(4-\alpha)} x^{3-\alpha}\right) ; \quad \mathfrak{D}^{\alpha} \Theta(x)=\left(\frac{\Gamma(2)}{\Gamma(2-\alpha)} x^{1-\alpha}, \frac{\Gamma(3)}{\Gamma(3-\alpha)} x^{2-\alpha}\right) .
$$

Now, by letting

$$
\eta_{1,2}(x, \xi)=\left(\frac{x-\xi}{2}, \frac{x-\xi}{2}\right), \quad \xi=0
$$

we have

$$
\eta_{1}(x, \xi) \cdot \mathfrak{D}^{\alpha} \Psi(x)=\frac{x^{3-\alpha}}{\Gamma(3-\alpha)}+\frac{3 x^{4-\alpha}}{\Gamma(4-\alpha)} ; \eta_{2}(x, \xi) \cdot \mathfrak{D}^{\alpha} \Theta(x)=\frac{x^{2-\alpha}}{2 \Gamma(2-\alpha)}+\frac{x^{3-\alpha}}{\Gamma(3-\alpha)} .
$$

Consider $\rho_{1}=\rho_{2}=1, x \in[0,1]$ and $\vartheta(x, \xi)=x^{2}-\xi$, therefore, we obtain

$$
\|\vartheta(x, \xi)\|^{2}=x^{4}, \quad \xi=0
$$


TABLE 1. Fractional multi-objective function, $k_{v}=0$

\begin{tabular}{||lcc||}
\hline$(\alpha)$ & Eq. (3.3) & Eq.(3.4) \\
\hline \hline 0.25 & 1.6 & 1.9 \\
\hline \hline 0.5 & 2.6 & 2.4 \\
\hline \hline 0.75 & 3.1 & 3.2 \\
\hline \hline
\end{tabular}

It is clear that

$$
\Psi(\xi)=\Psi(0)=(0,0) ; \quad \Theta(\xi)=\Theta(0)=(0,0)
$$

then by assuming

$$
\phi_{1}(\Psi(x)-\Psi(\xi))=5 x, \quad \phi_{2}(\Theta(x)-\Theta(\xi))=-5 x, \quad x \in[0,1]
$$

we conclude that

$$
\begin{aligned}
\eta_{1}(x, \xi) \cdot \mathfrak{D}^{\alpha} \Psi(x)+\rho_{1}\|\vartheta(x, \xi)\|^{2} & =\frac{x^{3-\alpha}}{\Gamma(3-\alpha)}+\frac{3 x^{4-\alpha}}{\Gamma(4-\alpha)}+x^{4} \\
& <5 x, \quad x \in[0,1] \\
& =\phi_{1}(\Psi(x)-\Psi(\xi))
\end{aligned}
$$

and

$$
\begin{aligned}
\eta_{2}(x, \xi) \cdot \mathfrak{D}^{\alpha} \Theta(x)+\rho_{2}\|\vartheta(x, \xi)\|^{2} & =\frac{x^{2-\alpha}}{2 \Gamma(2-\alpha)}+\frac{x^{3-\alpha}}{\Gamma(3-\alpha)}+x^{4} \\
& <5 x, \quad x \in[0,1] \\
& =-\phi_{2}(\Theta(x)-\Theta(\xi))
\end{aligned}
$$

Hence, the couple $(\Psi, \Theta)$ is $(\alpha, \rho, \eta, \vartheta)$-type univex at $\xi \in[0,1]$. Table 1 shows that for various values of $\alpha \in(0,1)$, the outcomes yield the fractional univexty of the couple $(\Psi, \Theta)$.

To apply the conditions of Theorem 3.1, we assume that $c_{1}=c_{2}=1$; thus, we have $c_{1} \rho_{1}+c_{2} \rho_{2}=2>0$ with the inequalities (3.3) and (3.4). This leads to all the conditions of Theorem 3.1 are achieved and hence, $\xi=0$ is an efficient solution. Note that if we let $\phi_{1}(Y)=3 Y$ and $\phi_{2}(Y)=-3 Y$, the couple $(\Psi, \Theta)$ is not $(\alpha, \rho, \eta, \vartheta)$-type univex at $\xi \in[0,1]$.

4.2. Case (ii) $k_{v}=1$. To evaluate the fractional Dunkl operator, a calculation implies that

$$
\sigma_{x^{2}}=x^{2}-2 \frac{v \cdot x^{2}}{v \cdot v}=-x^{2}, \quad \sigma_{x^{3}}=-x^{3} .
$$

Therefore, one can attain

$$
\eta_{1}(x, \xi) \cdot \mathfrak{D}^{\alpha} \Psi(x)=\frac{x^{3-\alpha}}{\Gamma(3-\alpha)}+\frac{x\left(2 x^{2}\right)^{\alpha}}{2}+\frac{3 x^{4-\alpha}}{\Gamma(4-\alpha)}+\frac{x\left(2 x^{3}\right)^{\alpha}}{2}
$$


and

$$
\eta_{2}(x, \xi) \cdot \mathfrak{D}^{\alpha} \Theta(x)=\frac{x^{2-\alpha}}{2 \Gamma(2-\alpha)}+\frac{x(2 x)^{\alpha}}{2}+\frac{x^{3-\alpha}}{\Gamma(3-\alpha)}+\frac{x\left(2 x^{2}\right)^{\alpha}}{2} .
$$

Table 2 shows the evaluation of the fractional multi-objective functions for different values of $\alpha$.

TABLE 2. Fractional multi-objective function, $k_{v}=1$

\begin{tabular}{||ccc||}
\hline$(\alpha)$ & Eq.(3.3) & Eq. (3.4) \\
\hline \hline 0.25 & 2.7 & 2.9 \\
\hline \hline 0.5 & 5 & 3.8 \\
\hline \hline 0.75 & 4.7 & 4.8 \\
\hline \hline
\end{tabular}

Thus, we conclude that the conditions of Theorem 3.1 are satisfied when $c_{1}=c_{2}=1$; such that $c_{1} \rho_{1}+c_{2} \rho_{2}=$ $2>0$ with the inequalities (3.3) and (3.4). Consequently, we obtain $\xi=0$ is an efficient solution.

4.3. Case (iii) $k_{v}=2$. By applying (1.2), we have

$$
\eta_{1}(x, \xi) \cdot \mathfrak{D}^{\alpha} \Psi(x)=\frac{x^{3-\alpha}}{\Gamma(3-\alpha)}+x\left(2 x^{2}\right)^{\alpha}+\frac{3 x^{4-\alpha}}{\Gamma(4-\alpha)}+x\left(2 x^{3}\right)^{\alpha}
$$

and

$$
\eta_{2}(x, \xi) \cdot \mathfrak{D}^{\alpha} \Theta(x)=\frac{x^{2-\alpha}}{2 \Gamma(2-\alpha)}+x(2 x)^{\alpha}+\frac{x^{3-\alpha}}{\Gamma(3-\alpha)}+x\left(2 x^{2}\right)^{\alpha} .
$$

Table 3 shows the evaluation of the fractional multi-objective functions for different values of $\alpha$. It is clear that the couple $(\Psi, \Theta)$ is not $(\alpha, \rho, \eta, \vartheta)$-type univex at $\xi \in[0,1]$. It is of $(\alpha, \rho, \eta, \vartheta)$-type univex at $\xi \in[0,1]$, when $\alpha \in(0,0.25]$. Hence, Theorem 3.1 can be applied only for this value of $\alpha$.

TABLE 3. Fractional multi-objective function, $k_{v}=2$

\begin{tabular}{||lcc||}
\hline$(\alpha)$ & Eq. (3.3) & Eq. (3.4) \\
\hline \hline 0.25 & 3.5 & 4.1 \\
\hline \hline 0.5 & 5.4 & 5.2 \\
\hline \hline 0.75 & 6.4 & 5.5 \\
\hline \hline
\end{tabular}

\section{Conclusion}

This effort is generalized, for the first time, two important concepts in science. The Dunkl operator and the Univex function, by utilizing the Riemann-Liouville fractional differential operator. These two generalizations are combined to deliver the fractional multi-objective problems. We studied the duality cases by minimize and maximize the desired function in the $\mathbb{R}^{n}$. Simulation is provided to apply the existing solutions. It has been found that the fractional case converges to the ordinary case. These problems can be employed in many studies not only in mathematics, but also in the economy; such as the utility function 
the cost function and the entropy function. One can replace the Riemann-Liouville fractional differential operator of any type of fractional calculus.

\section{ACKNOWLEDGEMENT}

The work here is partially supported by UKM grant: GUP-2017-064.

\section{REFERENCES}

[1] K.M. Kolwankar, A.D. Gangal, Fractional differentiability of nowhere differentiable functions and dimensions, Chaos 6 (4) (1996), 505-513.

[2] A.A. Kilbas, H.M. Srivastava, J.J. Trujiilo, Theory and Applications of Fractional Differential Equations. Amsterdam, Netherlands: Elsevier, 2006.

[3] G. Jumarie, Maximum entropy, information without probability and complex fractals: classical and quantum approach (Vol. 112). Springer Science \& Business Media, 2013.

[4] X-Jun Yang, D. Baleanu and H. M. Srivastava, Local Fractional Integral Transforms and Their Applications, Elsevier Ltd, 2016.

[5] X.-Jun. Yang, Local Fractional Functional Analysis and Its Applications, Asian Academic Publisher Limited, Hong Kong, 2011.

[6] C.F. Dunkl, Differential-difference operators associated to reflection groups, Trans. Amer. Math. Soc. 311 (1989), $167-183$.

[7] M.Rosler, M. Voit, Markov processes related with Dunkl operators. Adv. Appl. Math. 21(4)(1998), 575-643.

[8] R. W. Ibrahim, Optimality and duality defined by the concept of tempered fractional univex functions in multi-objective optimization, Int. J. Anal. Appl. 15 (2017), 75-85.

[9] R. W. Ibrahim, Abdullah Gani, A new algorithm in cloud computing of multi-agent fractional differential economical system, Computing 98 (11) (2016), 1061-1074.

[10] R. W. Ibrahim, Hamid A. Jalab, and Abdullah Gani, Perturbation of fractional multi-agent systems in cloud entropy computing, Entropy 18 (2016), 31.

[11] R. W. Ibrahim, Abdullah Ghani, Hybrid cloud entropy systems based on Wiener process, Kybernetes 45 (7) (2016), 1072-1083.

[12] R. W. Ibrahim, and Abdullah Gani, A mathematical model of cloud computing in the economic fractional dynamic system, Iran. J. Sci. Technol. Trans. A, Sci. 42 (2018), 65-72. 